ADDENDUM

doi:10.1038/nature13075

\title{
Addendum: Enterotypes of the human gut microbiome
}

Manimozhiyan Arumugam, Jeroen Raes, Eric Pelletier, Denis Le Paslier, Takuji Yamada, Daniel R. Mende, Gabriel R. Fernandes, Julien Tap, Thomas Bruls, Jean-Michel Batto, Marcelo Bertalan, Natalia Borruel, Francesc Casellas, Leyden Fernandez, Laurent Gautier, Torben Hansen, Masahira Hattori, Tetsuya Hayashi, Michiel Kleerebezem, Ken Kurokawa, Marion Leclerc, Florence Levenez, Chaysavanh Manichanh, H. Bjørn Nielsen, Trine Nielsen, Nicolas Pons, Julie Poulain, Junjie Qin, Thomas Sicheritz-Ponten, Sebastian Tims, David Torrents, Edgardo Ugarte, Erwin G. Zoetendal, Jun Wang, Francisco Guarner, Oluf Pedersen, Willem M. de Vos, Søren Brunak, Joel Doré, MetaHIT Consortium, Jean Weissenbach, S. Dusko Ehrlich \& Peer Bork

Nature 473, 174-180 (2011); doi:10.1038/nature09944 and corrigendum 474, 666 (2011); doi:10.1038/nature10187

It has been drawn to our attention that the methods described in the main text and the Supplementary Information of this Article have been considered by some researchers to be insufficient to enable them to identify enterotypes in their own data sets. Enterotypes were originally defined in this Article (page 177) as "densely populated areas in a multi-dimensional space of community composition" and should not be seen as discrete clusters, but as a way of stratifying samples to reduce complexity. Additionally, the Fig. 2 legend should not imply that between-class analysis is simply a method of visualizing principal component analysis (PCA); rather, it is a supervised rather than an unsupervised analysis of data because it incorporates the outcome of clustering of data. To simplify enterotype identification in the original and other data sets, we have developed a comprehensive tutorial at http://enterotype.embl.de-which is a website on enterotypes that will be updated as methods improve. We thank Ivica Letunic and Paul Costea from EMBL for setting up the tutorial. 